\title{
Over-Expression of MicroRNA-22 Protects Cardiomyocytes from Ischemic Injury
}

\author{
Lin Ling ${ }^{*}$, Zicheng Ling2, Shaohua $\mathrm{Gu}^{3}$ \\ ${ }^{1}$ Department of Cardiology, The First Affiliated Hospital of Soochow University, Suzhou, China \\ ${ }^{2}$ Shanghai University of Traditional Chinese Medicine, Shanghai, China \\ ${ }^{3}$ Department of Nephrology, The Third People’s Hospital of Kunshan, Suzhou, China \\ Email: *linglin@suda.edu.cn
}

How to cite this paper: Ling, L., Ling, Z.C. and $\mathrm{Gu}$, S.H. (2019) Over-Expression of MicroRNA-22 Protects Cardiomyocytes from Ischemic Injury. World Journal of Cardiovascular Diseases, 9, 737-745. https://doi.org/10.4236/wjcd.2019.910066

Received: August 28, 2019

Accepted: October 15, 2019

Published: October 18, 2019

Copyright () 2019 by author(s) and Scientific Research Publishing Inc. This work is licensed under the Creative Commons Attribution International License (CC BY 4.0).

http://creativecommons.org/licenses/by/4.0/

(c) (i) Open Access

\begin{abstract}
Objective: To investigate the protection effect of over-expressed MicroRNA22 on ischemic cardiomyocyte. Methods: Cardiomyocytes cultured in hypoxia and ischemia condition were transfected with recombinant adenovirus vector containing microRNA-22 plus a recombinant green fluorescent protein (GFP). Transduction efficiency and expression of microRNA-22 were assessed. The expression of downstream PTEN protein expression was detected. After successful transduction, cell activities including viability, nuclei proliferation and cell apoptosis were assessed. We used CCK-8 assay to assess cell activities, EdU assay to assess nuclei proliferation and Caspase-3/LDH assay to assess cell apoptosis. Results: MicroRNA-22 was transfected successfully using adeno-virus. The expression of microRNA-22 was significantly increased. The transduction efficiency reached more than 95\%. Over-expression of microRNA-22 in cardiomyocytes down regulated PTEN protein level. Overexpression of microRNA-22 increased cell activities, DNA proliferation and decreased cell apoptosis. Conclusion: Over-expression of microRNA-22 protected cardiomyocytes from ischemic injury.
\end{abstract}

\section{Keywords}

MicroRNA-22, Cardiomyocyte, Cell Activities

\section{Introduction}

Acute myocardial infarction is a myocardial ischemic disease which seriously endangering people's lives [1]. After myocardial infarction, a large number of cardiomyocytes appear apoptosis, necrosis, autophagy or dysfunction due to ischemia, hypoxia and cell metabolism disorders [2] [3] [4] [5]; long-term left ventricular pressure overload eventually leads to ventricular dilatation and heart 
failure [6] [7]. After acute myocardial infarction, reducing myocardial cell apoptosis, delaying ventricular remodeling to improve cardiac function is the key to myocardial repair [8] [9].

Recent studies have shown that microRNAs play an important regulatory role in human or animal heart disease, such as myocardial ischemia and cardiac hypertrophy [10] [11]. MicroRNA-22 is a member of the newly discovered microRNA family, widely expressed in myocardium and skeletal muscle [12] [13]. In the TAC pressure over-load model, microRNA-22 knockout mice showed more significant de-compensated cardiac systolic function, and the degree of cardiac dilatation was increased. The expression of Dystrophin, Ldb3, Mlp and Titin which encoded myocardium skeleton protein was decreased. At the same time, the positive inotropic effect on dobutamine disappeared and the $\mathrm{Ca}^{2+}$ transient current in cytoplasm decreased, while $\mathrm{Ca}^{2+}$ dependent ATPase activity decreased. These suggested that microRNA-22 was important for normal cell structure maintenance and $\mathrm{Ca}^{2+}$ current stability under pressure over-load [14] [15]. At present, the protective effect of microRNA-22 on ischemic and hypoxic cardiomyocytes has not been reported.

In this study, we used adenovirus vector over-expressing microRNA-22 to transfect cardiomyocytes cultured under hypoxia condition. We aimed to observe the protective effect of over-expressed microRNA-22 on hypoxic cardiomyocytes in vitro, providing theoretical and practical basis for microRNA-22 treatment for myocardial repair after infarction.

\section{Materials and Methods}

\subsection{Experimental Animals}

Ethics Statement Animal study were approved by Soochow University Scientific and Animal Ethics Committee (Approval Number: 20150086) and were in compliance with the Chinese national regulations on the use of experimental animals. Procedures for animal studies were performed in accordance with the Guide for the Care and Use of Laboratory Animals published by the US National Institutes of Health (US National Institutes of Health; National Institutes of Health publication no. 85-23, revised 1996). We used SPF grade C57/BL6 male suckling mouse aged 1-3d in our experiment. All animals were purchased from the laboratory animal center of Soochow University. They were maintained on standard diet and water at a 12/12 light/dark cycle at the animal center of the first affiliated hospital of soochow university.

\subsection{Cardiomyocytes Culture and Hypoxic Model Construction}

Mouse was sacrificed by euthanasia with carbon dioxide. Cardiomyocytes were isolated from hearts by adding $0.25 \%$ trypsin (Invitrogen; Thermo Fisher Scientific, Inc., Waltham, MA, USA)/0.02\% EDTA (Invitrogen; Thermo Fisher Scientific, Inc., Waltham, MA, USA) for digestion and the digestive fluid was collected by centrifugation. After centrifugation, the cells were inoculated in $25 \mathrm{~cm}^{2}$ culture flask and cultured in $37^{\circ} \mathrm{C}, 5 \% \mathrm{CO}_{2}$ saturated humidity incubator (Ther- 
mo). For construction of hypoxia condition, cardiomyocytes were incubated for $24 \mathrm{~h}$ with $1 \%$ fetal bovine serum (Gibco; Thermo Fisher Scientific, Inc.) and then transferred to hypoxia buffer (Gibco; Thermo Fisher Scientific, Inc.) and cultured in a saturated $\left(95 \% \mathrm{~N}_{2}+5 \% \mathrm{CO}_{2}\right)$ container at $37^{\circ} \mathrm{C}$.

\subsection{MicroRNA-22 Over-Expressed Adenovirus Vector Transfection}

Recombinant adenovirus vector containing microRNA-22 plus a recombinant GFP (Adeno-miR22) or GFP only (Adeno-null) were produced by GenScript Company (Nanjing, China). Cardiomyocytes were cultured to $70 \%$ confluency. The supernatant was reduced to $800 \mathrm{~mL}$, and purified adenoviral vector was added to the medium at multiplicities of infection of 10 to 100 . After incubation at $37^{\circ} \mathrm{C}, 5 \% \mathrm{CO}_{2}$ for $2 \mathrm{~h}, 1200 \mathrm{~mL}$ complete medium was added and cells were cultured for $12 \mathrm{~h}$. Next, virus-containing medium was removed and replaced with fresh medium. Transduction efficiency and expression of microRNA-22 were detected by Taqman RT-PCR. Primers were designed by Invitrogen company. RNA extraction and PCR reaction process were conducted according to the manufacturer's construction. Gene expression was assessed as $\Delta \Delta \mathrm{Ct}=(\mathrm{sam}$ ple Ct miR-22 - sample CtU6 mRNA) - (control Ct miR-22 - control Ct U6 mRNA). The fluorescence intensity of GFP was detected by flow cytometry (Thermo) and fluorescence microscopy (ZEISS). The expression of microRNA-22 and downstream PTEN protein expression were detected. The experimental cells were divided into two groups including Adeno-miR22 group and Adeno-null group.

\subsection{Cell Viability Assessment}

Cell viability was assessed using a CCK-8 assay, based on the enzymatic reduction of WST- 8 in living cells and the production of a proportional color change. Briefly, $1 \times 10^{4}$ cells suspended in $150 \mu \mathrm{l}$ complete medium were seeded in each well of a 96-well culture plate and incubated at $37^{\circ} \mathrm{C}$ under $5 \% \mathrm{CO}_{2}$ for $24 \mathrm{~h}$. The WST- 8 cell proliferation reagent $(50 \mu \mathrm{l})$ was added and incubated for $4 \mathrm{~h}$ under $5 \% \mathrm{CO}_{2}$ at $37^{\circ} \mathrm{C}$. The negative control comprised WST- 8 reagent and complete medium with no cells. The absorbance was measured at $450 \mathrm{~nm}$ using a spectrophotometer (Huier Company, Hangzhou, China) and the optical density (OD) was calculated as $\mathrm{OD}=$ ODsample - ODcontrol. Each experiment was performed in triplicate wells and repeated three times.

\subsection{Cell DNA Synthesis Ability Determination}

DNA synthesis was measured using Promega's Click-iT ${ }^{\circledR}$ EdU kit. Briefly, the cells cultured in 6-well plates were incubated with EdU for 24 h. Following fixation and permeabilization, $0.5 \mathrm{ml} \mathrm{Click-iT^{ \circledR }}$ reaction cocktail was added and incubated for $30 \mathrm{~min}$ at room temperature in the dark. Hoechst 33342 (Invitrogen, USA) was used to stain the cell nuclei. The cells were observed using a fluorescence microscope, and nuclei undergoing DNA synthesis were stained red. The numbers of proliferating nuclei were counted in 10 randomly selected fields 
(magnification, $\times 100$ ) and the average was calculated. The rate of DNA synthesis was calculated as the number of proliferating nuclei/100. Each experiment was performed in triplicate wells and repeated three times.

\subsection{Detection of Cell Apoptosis}

Cell apoptosis was assessed by caspase- 3 activity. The activity of caspase-3 was determined using the caspase-3 activity kit (Beyotime Institute of Biotechnology, Shanghai, China). Cell lysates were prepared after their respective treatment with various designated treatments. Assays were performed on 96-well microtitre plates by incubating $10 \mu \mathrm{l}$ protein of cell lysate per sample in $80 \mu \mathrm{l}$ reaction buffer (1\% NP-40, $20 \mathrm{mM}$ Tris-HCl (PH 7.5), $137 \mathrm{mM} \mathrm{Nad}$ and 10\% glycerol) containing $10 \mu \mathrm{l}$ caspase-3 substrate (Ac-DEVD-pNA) $(2 \mathrm{mM})$. Lysates were incubated at $37^{\circ} \mathrm{C}$ for $4 \mathrm{~h}$. Samples were measured with an ELISA reader at an absorbance of $405 \mathrm{~nm}$. The detail analysis procedure was described in the manufaturer's protocol. To further measure the extent of cell injury, the LDH activity was also tested. At the end of the incubation period, the culture supernatants were collected. The activity of LDH was detected at $490 \mathrm{~nm}$ according to the LDH assay kit (Beyotime Institute of Biotechnology, Shanghai, China).

\subsection{Western Blot Analysis}

The harvested cells were lysed with RIPA lysis buffer and protease inhibitor cocktail. Protein concentrations were quantified using a BCA protein assay kit. 30 ug proteins were separated using 10\% SDS-polyacrylamide gel electrophoresis and transferred onto a PVDF membrane. The membrane was blocked with Tris-buffered saline-Tween 20 (TBS-T) and 5\% nonfat dried milk for $2 \mathrm{~h}$, and incubated with primary antibody (mouse monoclonal to PTEN, 1:1000, Cat No. AB32199, abcam, Cambridge, UK) overnight at $4^{\circ} \mathrm{C}$. Following washing twice with TBS-T and incubation with peroxidase-conjugated secondary antibodies (goat anti mouse 1:1000, A996702 Amyjet Scientific, China) for $1 \mathrm{~h}$ at room temperature, the bands were detected using a chemiluminescence detection system (Invitrogen, USA). The band intensities were quantified using the Photo-Image System (Siemens, Germany).

\section{Statistical Processing}

Data were presented as mean \pm standard deviation and were analyzed using SPSS 22.0 statistical software (SPSS, Inc., Chicago, IL, USA). Comparisons between two groups were performed using an unpaired Student's t test. $P<0.05$ was considered to indicate a statistically significant difference.

\section{Results}

\subsection{Adenovirus Mediated Over-Expression of microRNA-22 in Cardiomyocytes Down-Regulated PTEN Expression}

Adenovirus mediated over-expression of microRNA-22 in cardiomyocytes co- 
expressed GFP. The ratio of GFP positive cells was detected by flow cytometry, indicating that more than $95 \%$ cells were successfully transfected with GFP (Figure 1). The expression of microRNA-22 was confirmed by qRT-PCR after adenovirus transfection. The results showed that the expression of microRNA22 was significantly increased by 10.75 times compared with that of adeno-null group, ${ }^{*} p<0.05$. This suggested that adenovirus transfection with high expression of microRNA-22 was successful (Figure 2). Western blot showed that the protein expression of PTEN was down-regulated after high expression of microRNA-22 (Figure 3).

\subsection{DNA Synthesis Assessment}

After adenovirus transfection, EdU was used to detect the proliferation of nuclei in both groups, which represented nuclei proliferation. Red represented for positive nuclei and blue for all nuclei. Compared with adeno-null group, the nuclei proliferation in adeno-miR22 group was significantly increased, ${ }^{\star} p<0.05$ (Figure 4).

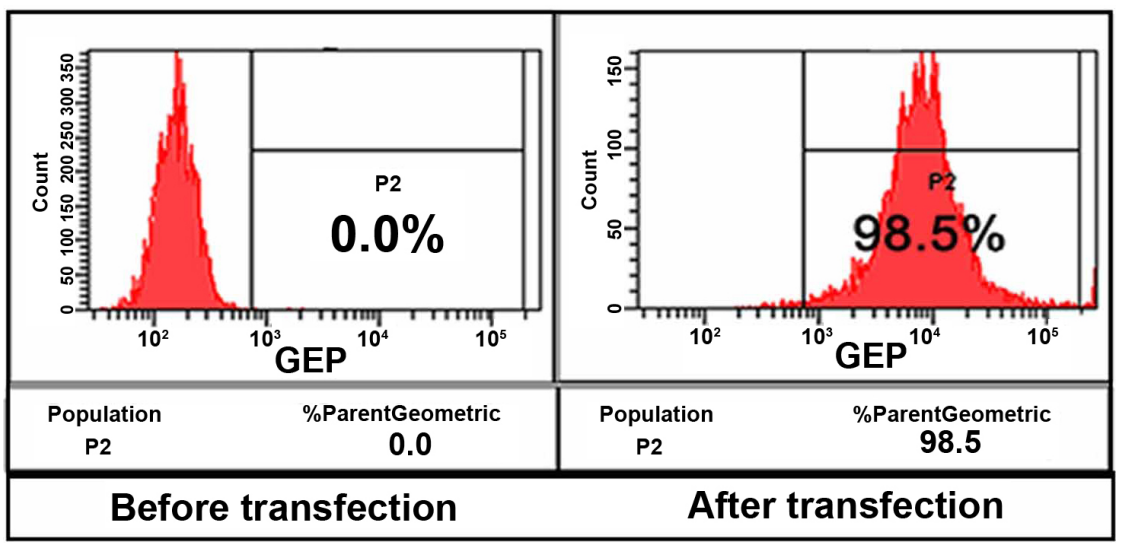

Figure 1. GFP positive rate of more than $95 \%$ in cardiomyocytes after adenovirus transfection.

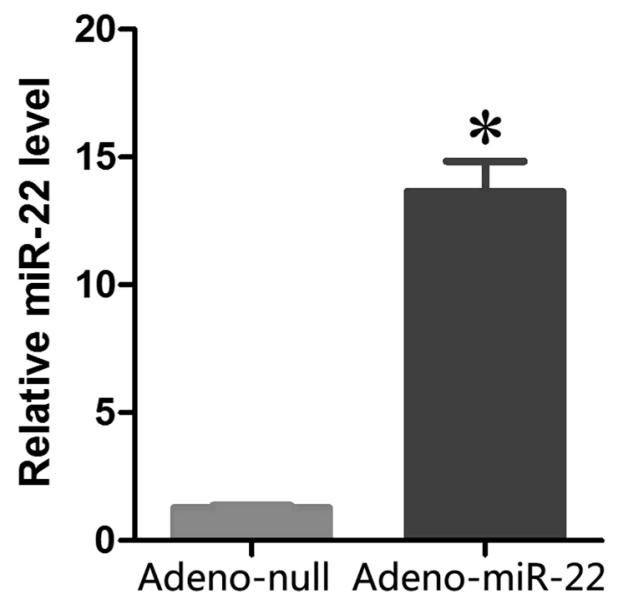

Figure 2. MicroRNA-22 expression was significantly increased after adenovirus transfection, ${ }^{\star} p<0.05$ vs. adeno-null group. 


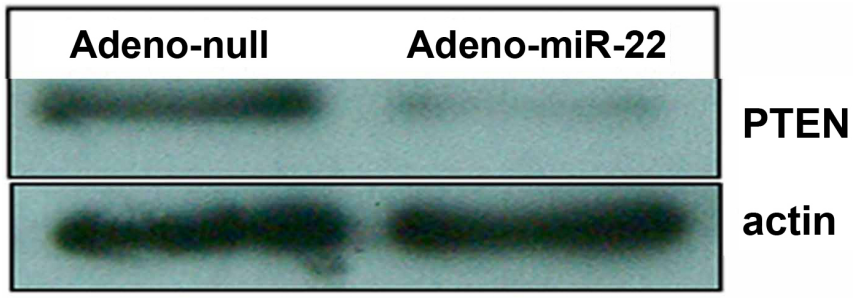

Figure 3. High expression of microRNA-22 down-regulated PTEN protein expression.

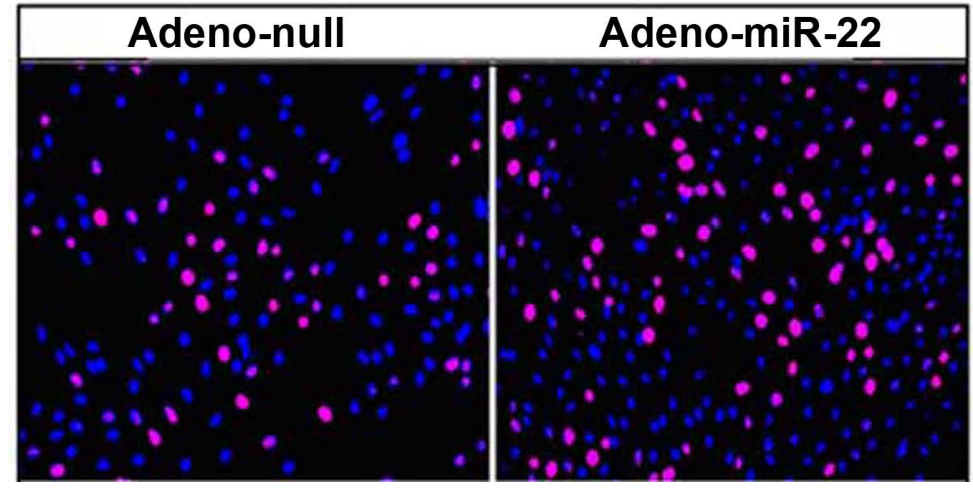

Figure 4. Increased nuclei proliferation in cells transfected with adeno-miR22.

\subsection{Observation of Cytological Characteristics}

The cell growth curve recorded. Cell viability was measured by CCK-8 assay. Apoptosis was detected by Caspase-3/LDH assay. Compared with adeno-null group, the cells with high expression of microRNA-22 showed increased cell growth velocity and viability as well as decreased cell apoptosis, ${ }^{\star} p<0.05$ vs. adeno-null group (Figures 5(A)-(D)).

\section{Discussion}

Acute myocardial infarction leads to ventricular dilatation and heart failure [6] [7]. In addition to traditional drugs, explore new methods of myocardial repair in the field of molecular medicine is the current research focus.

Recent studies have shown that microRNAs play an important regulatory role in human or animal heart disease, such as myocardial ischemia and cardiac hypertrophy. The microRNA is an endogenous non-protein-encoding RNA of about $21-23$ nucleotides. It is important to regulate the translational efficiency or stability of the target gene mRNA by identifying the 3 'end of the target gene mRNA. microRNAs are involved in regulating various physiological and pathological processes, including cell development, differentiation, metabolism, growth, proliferation and apoptosis [10] [11]. At present, microRNAs have been found to be closely related to myocardial repair after myocardial ischemia. Researches showed that in the model of myocardial ischemia/reperfusion injury, the infarct size of microRNA-494 over-expressing transgenic mice was significantly decreased and the cardiac function was significantly improved compared with the control group. The mechanism was as follows: inhibition of pro-apoptotic genes 
(A)

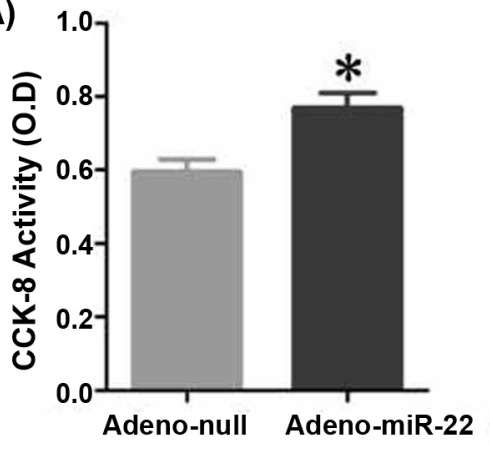

(C)

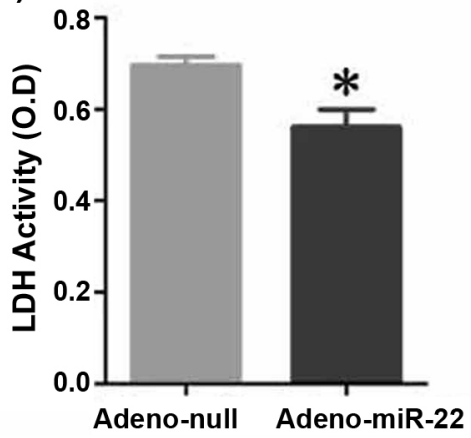

(B)

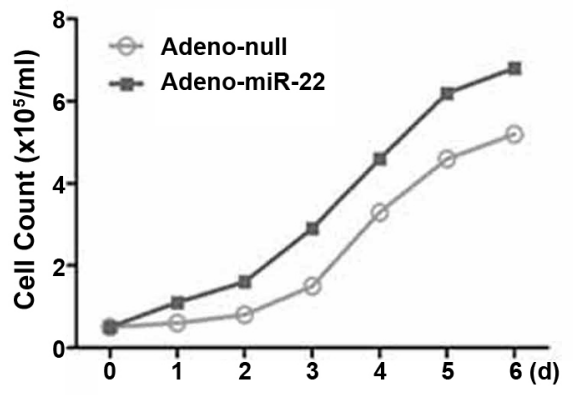

(D)

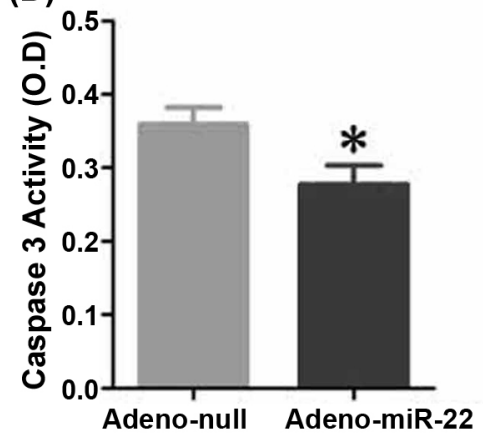

Figure 5. Over expression of microRNA-22 promoted cardiomyocyte viability, reduced cell apoptosis, ${ }^{\star} p<0.05$ vs. adeno-null group.

PTEN, ROCK1 and CaMKII $\delta$ expression, activation of PI3K/AKT pathway, reduce Caspase-3 activity and LDH release, thereby promoting cell survival and reduce apoptosis. This confirmed that in the model of myocardial ischemia/reperfusion injury, the use of exogenous gene transfer method to regulate microRNA can repair damaged myocardium and improve cardiac function [12]. Another study found that after myocardial ischemia, over-expression of microRNA1 can promote cardiomyocyte apoptosis, with the mechanism of inhibiting anti-apoptotic gene Bcl-2, IGF-1 expression and inducing cells into the process of apoptosis. Over-expression of microRNA-21 can reduce cell apoptosis, and its mechanism is to interfere with the activity of PDCD4 and AP-1, inhibit the activity of PTEN and SP-1, activate ERK-MAPK pathway, and promote cell survival and cytokine secretion, mediating cardiac function improvement after myocardial infarction [16] [17].

MicroRNA-22 is widely expressed in the myocardium and skeletal muscle as a member of the newly discovered microRNA family [13] [14]. MicroRNA-22 is also involved in the regulation of cell survival and apoptosis. In the in vitro brain aging model, high expression of microRNA-22 can reduce the degradation of neuronal cells and enhance the activity of neuronal cells. It can protect neurons and reduce ischemia-reperfusion injury [18] [19].

In this study, we found that the over-expression of microRNA-22 in cultured cardiomyocytes in vitro increased cardiomyocytes' viability, cell proliferation 
and reduced hypoxia-induced apoptosis. It was confirmed that microRNA-22 could protect cardiac cells under hypoxia conditions. Further analysis showed that high expression of microRNA-22 down-regulated PTEN protein expression, which is involved in a number of cell-related pathways in the regulation of cell survival and apoptosis process. This suggested that the cytoprotective effect of microRNA-22 may be achieved by modulating PTEN. The limitation of this work is that the longer effect of microRNA-22 on myocardium could be observed. Our future work may focus on this point.

\section{Conclusion}

Over-expressing of microRNA-22 in cardiomyocytes can increase cardiomyocyte activity, decrease hypoxia-induced cell apoptosis and reduce myocardial cell hypoxia injury.

\section{Funding}

The present study was supported by the Youth Science and Technology of Suzhou Science and Education Project (grant No. KJXW2013004), and the Science Foundation for Youth Teacher of Soochow University (grant No. SDY2013A29).

\section{Conflicts of Interest}

The authors declare no conflicts of interest regarding the publication of this paper.

\section{References}

[1] Mozaffarian, D., Benjamin, E.J., Go, A.S., et al. (2015) Heart Disease and Stroke Statistics-2015 Update: A Report from the American Heart Association. Circulation, 131, e29-e322.

[2] Huttin, O., Coiro, S., Selton-Suty, C., et al. (2016) Prediction of Left Ventricular Remodeling after a Myocardial Infarction: Role of Myocardial Deformation: A Systematic Review and Meta-Analysis. PLoS ONE, 11, e0168349. https://doi.org/10.1371/journal.pone.0168349

[3] Shah, A.M., Hung, C.L., Shin, S.H., et al. (2011) Cardiac Structure and Function, Remodeling, and Clinical Outcomes among Patients with Diabetes after Myocardial Infarction Complicated by Left Ventricular Systolic Dysfunction, Heart Failure, or Both. American Heart Journal, 162, 685-691.

https://doi.org/10.1016/j.ahj.2011.07.015

[4] Dhalla, N.S., Rangi, S., Babick, A.P., Zieroth, S. and Elimban, V. (2012) Cardiac Remodeling and Subcellular Defects in Heart Failure Due to Myocardial Infarction and Aging. Heart Failure Reviews, 17, 671-681. https://doi.org/10.1007/s10741-011-9278-7

[5] Manrique, A., Lemarchand, P., Delasalle, B., et al. (2016) Predictors of Ventricular Remodelling in Patients with Reperfused Acute Myocardial Infarction and Left Ventricular Dysfunction Candidates for Bone Marrow Cell Therapy: Insights from the BONAMI Trial. European Journal of Nuclear Medicine and Molecular Imaging, 43, 740-748. https://doi.org/10.1007/s00259-015-3279-Z

[6] Huttin, O., Mandry, D., Eschalier, R., et al. (2017) Cardiac Remodeling Following 
Reperfused Acute Myocardial Infarction Is Linked to the Concomitant Evolution of Vascular Function as Assessed by Cardiovascular Magnetic Resonance. Journal of Cardiovascular Magnetic Resonance, 19, 2-4. https://doi.org/10.1186/s12968-016-0314-6

[7] Ertl, G., Brenner, S. and Angermann, C.E. (2017) Cardiac Remodeling after Myocardial Infarction: Clinical Practice Update. Herz, 42, 107-120.

https://doi.org/10.1007/s00059-016-4530-5

[8] Roubille, F. and Lacampagne, A. (2014) New Drug Avenues for Cardioprotection in Patients with Acute Myocardial Infarction. American Journal of Cardiovascular Drugs, 14, 73-77. https://doi.org/10.1007/s40256-013-0049-9

[9] Song, M., Jang, H., Lee, J., et al. (2014) Regeneration of Chronic Myocardial Infarction by Injectable Hydrogels Containing Stem Cell Homing Factor SDF-1 and Angiogenic Peptide Ac-SDKP. Biomaterials, 35, 2436-2445. https://doi.org/10.1016/j.biomaterials.2013.12.011

[10] Bauters, C. and Pinet, F. (2016) MicroRNAs as Circulating Biomarkers of Left Ventricular Remodeling after Myocardial Infarction. Cardiology, 133, 262-263. https://doi.org/10.1159/000443204

[11] Martinez-Fernandez, A. (2014) MicroRNA Therapy for the Failing Heart. Circulation, 7, 393-394. https://doi.org/10.1161/CIRCGENETICS.114.000687

[12] Wang, X., Zhang, X., Ren, X.P., et al. (2010) MicroRNA-494 Targeting Both ProApoptotic and Anti-Apoptotic Proteins Protects against Ischemia/Reperfusion-Induced Cardiac Injury. Circulation, 122, 1308-1318. https://doi.org/10.1161/CIRCULATIONAHA.110.964684

[13] Xiong, J. (2012) Emerging Roles of MicroRNA-22 in Human Disease and Normal Physiology. Current Molecular Medicine, 12, 247-258. https://doi.org/10.2174/156652412799218886

[14] Song, S.J., Ito, K., Ala, U., et al. (2013) The Oncogenic MicroRNA miR-22 Targets the TET2 Tumor Suppressor to Promote Hematopoietic Stem Cell Self-Renewal and Transformation. Cell Stem Cell, 13, 87-101. https://doi.org/10.1016/j.stem.2013.06.003

[15] Gurha, P., Abreu-Goodger, C., Wang, T., et al. (2012) Targeted Deletion of MicroRNA-22 Promotes Stress-Induced Cardiac Dilation and Contractile Dysfunction. Circulation, 125, 2751-2761. https://doi.org/10.1161/CIRCULATIONAHA.111.044354

[16] Ono, K., Kuwabara, Y. and Han, J. (2011) MicroRNAs and Cardiovascular Diseases. The FEBS Journal, 278, 1619-1633. https://doi.org/10.1111/j.1742-4658.2011.08090.x

[17] Chen, J. and Wang, D.Z. (2012) MicroRNAs in Cardiovascular Development. Journal of Molecular and Cellular Cardiology, 12, 949-957. https://doi.org/10.1016/j.yjmcc.2012.01.012

[18] Jovicic, A., Zaldivar Jolissaint, J.F., Moser, R., et al. (2013) MicroRNA-22 (miR-22) Overexpression Is Neuroprotective via General Anti-Apoptotic Effects and May also Target Specific Huntington's Disease-Related Mechanisms. PLoS ONE, 8, e54222. https://doi.org/10.1371/journal.pone.0054222

[19] Yu, H., Wu, M., Zhao, P., et al. (2015) Neuro-Protective Effects of Viral Over-Expression of MicroRNA-22 in Rat and Cell Models of Cerebral Ischemia-Reperfusion Injury. Journal of Cellular Biochemistry, 116, 233-241. https://doi.org/10.1002/jcb.24960 